\title{
Charge state dependence of channeled ion energy loss
}

\section{Citation}

Golovchenko, J. A., A. N. Goland, J. S. Rosner, C. E. Thorn, H. E. Wegner, H. Knudsen, and C. D. Moak. 1981. Charge State Dependence of Channeled Ion Energy Loss. Physical Review B 23, no. 3: 957-966. doi:10.1103/physrevb.23.957.

\section{Published Version}

doi:10.1103/PhysRevB.23.957

\section{Permanent link}

http://nrs.harvard.edu/urn-3:HUL.InstRepos:29407056

\section{Terms of Use}

This article was downloaded from Harvard University's DASH repository, and is made available under the terms and conditions applicable to Other Posted Material, as set forth at http:// nrs.harvard.edu/urn-3:HUL.InstRepos:dash.current.terms-of-use\#LAA

\section{Share Your Story}

The Harvard community has made this article openly available.

Please share how this access benefits you. Submit a story.

Accessibility 


\title{
Charge state dependence of channeled ion energy loss
}

\author{
J. A. Golovchenko \\ Bell Laboratories, Murray Hill, New Jersey 07974
}

\author{
A. N. Goland, J. S. Rosner, C. E. Thorn, and H. E. Wegner \\ Brookhaven National Laboratory, Upton, New York 11973 \\ H. Knudsen* and C. D. Moak \\ Oak Ridge National Laboratory, Oak Ridge, Tennessee 37830 \\ (Received 19 May 1980)
}

\begin{abstract}
The charge state dependence of channeled ion energy loss has been determined for a series of ions ranging from fluorine to chlorine along the $\langle 110\rangle$ direction in a silicon crystal. Energy losses for both bare ions and ions partially clothed with bound electrons at $E / A \cong 3 \mathrm{MeV} / \mathrm{amu}$ have been measured. The energy-loss rate for bare ions follows a strict $Z_{1}^{2}$ scaling and agrees reasonably well with quantal perturbation calculations without the need for polarization or Bloch corrections. An explanation for this result is discussed. The clothed-ion energy losses appear to demonstrate screening effects that agree qualitatively with simple estimates. The angular dependence of the observed energy-loss effects is also presented.
\end{abstract}

\section{INTRODUCTION}

The basic processes involved in slowing down fast heavy ions as they pass through solids are qualitatively understood. ${ }^{\prime}$ As an ion proceeds along its track, it interacts via the Coulomb force with target electrons transferring kinetic energy to electronic excitation of the target. If the atomic number $Z_{1}$, of the ion is low enough and or its velocity, $v$, high enough $\left(v \gg Z_{1}^{2 / 3} e^{2} / \hbar\right.$ for random penetration) it will proceed in a completely stripped state. Various attempts have been made to explain observed energyloss rates under these conditions through perturbation treatments. At the highest velocities first-order quantal perturbation calculations yield expressions with a $Z_{1}^{2}$ dependence in the stopping power which can accurately account for experimental results for both random ${ }^{2}$ and channeled ${ }^{3}$ motions in the target. For decreasing velocities terms with higher-order $Z_{1}$ dependence may be expected to become important. At the same time (as $v$ decreases towards $Z_{1}^{2 / 3} e^{2 / \hbar}$ ) the charge state of the ion will begin fluctuating due to electron capture and loss processes which results in a modification of the effective coupling between the ion and target.

Scientific interest in this problem remains alive for two reasons. First the charge state distribution for heavy ions inside solid targets is not known. ${ }^{4}$ Second, there has been renewed interest in deviations from simple first-order perturbation theory stopping power predictions (i.e., higher-order $Z_{1}$ effects).
Thus uncertainty about the importance of the latter effect coupled with the poor state of knowledge about the former has made the details of heavy ion stopping in solids mainly an empirical science.

In addition to the $Z_{1}^{2}$ dependence for stopping power, terms in higher orders of $Z_{1}$ have been found experimentally by Barkas ${ }^{5}$ and theoretical descriptions have been presented by Jackson and McCarthy ${ }^{6}$ and by Ashley, Brandt, and Ritchie: ${ }^{7}$ Recently Lindhard ${ }^{8}$ has pointed out that terms must exist in even powers of $Z_{1}$ as well as the odd power terms proposed earlier, and that the $Z_{1}^{3}$ term and the $Z_{1}^{4}$ term can be comparable in magnitude and opposite in sign. Measurements for stopping powers of protons, $\alpha$ particles, and $\mathrm{Li}$ ions have been reported by Andersen, Bak, Knudsen, Petersen, and Nielsen ${ }^{9}$ and these data indicate the presence of $Z_{1}^{3}$ and $Z_{1}^{4}$ terms in the stopping-power formulas. It would be desirable to carry out such measurements for ions of higher $Z_{1}$ but the aforementioned difficulty of charge state fluctuations tends to set in above $\mathrm{Li}$.

A major step towards alleviating this state of affairs was initiated recently in a series of experiments performed at the Oak Ridge National Laboratory (ORNL) ${ }^{10}$ It was demonstrated that under very strict channeling conditions, charge state integrity could be maintained, for certain trajectories and charge states, over the entire track as the ion passes through a thin crystal. More specifically the electron capture and loss cross sections were shown to be significantly reduced for ions traveling down the low 
electron density, central region of crystal channels. Such trajectories also have a signature feature in channeled energy-loss spectra in that they are associated with least energy loss (for given charge state) particles. Subsequently Datz et al. ${ }^{11}$ published results showing $Z_{1}^{3}$ effects as well as bound $K$ electron screening effects for channeled ions.

It was later pointed out that uncertainties in the interpretation of the least energy-loss portion of the spectrum existed due to energy-loss straggling effects. $^{12}$ Consequently certain conclusions, e.g., those pertaining to $Z_{1}^{3}$ stopping effects were thrown into doubt.

In the following we report on an extension of these charge state channeling energy-loss measurements. First we have extended the measurements to cover $Z_{1}$ values 9 to 17 . We believe the straggling effect referred to earlier is of no consequence here. In addition we have changed from gold to silicon targets. The absence of complications due to shell effects and the availability of theoretical channeling predictions for this target make it attractive. We have also studied the case of axial rather than planar channeling.

Finally we note that if the charge state of an ion could remain fixed while the ion loses energy, another interesting study is the comparison of the energy loss for a bare ion $\left(Z_{1}-1\right)$ with that of one electron ion $Z_{1}$, the two ions having the same total charge but a different spatial distribution of charge. It would be expected that as the ion swept through the slowing medium, those electrons lying far off the ion path would interact in the same way for the two ions but for electrons lying inside the column of average $K$ shell radius of the one-electron ion, a stronger interaction would occur for the $Z_{1}$ one electron ion than for the bare ion $Z_{1}-1$. This small difference in stopping power would be observable only if the ionic charge state could be preserved while the ion is slowing down.

\section{EXPERIMENTAL METHODS}

Figure 1 contains a schematic layout of the experiment. The Brookhaven National Laboratory (BNL) Tandem van de Graaff was used to accelerate beams of ${ }^{19} \mathrm{~F},{ }^{24} \mathrm{Mg},{ }^{28} \mathrm{Si},{ }^{32} \mathrm{~S}$, and ${ }^{35} \mathrm{Cl}$ to $3.086 \mathrm{MeV} / \mathrm{amu}$.

After energy analysis the beam was attenuated by a $0.2-\mathrm{mm}$ aperture and passed through a thin carbon foil ( 2 to $20 \mu \mathrm{g} / \mathrm{cm}^{2}$ ) to produce a distribution of charge states. The desired charge state for the measurement was selected by a $65^{\circ}$ deflection in the beam switching magnet and dispersed on a slit $( \pm 0.1$ $\mathrm{mm}$ ) in front of the scattering chamber containing a thin Si single crystal and goniometer. The goniometer was constructed so that the crystal could be removed from the beam, and energy losses were directly obtained as the difference in measured energy with

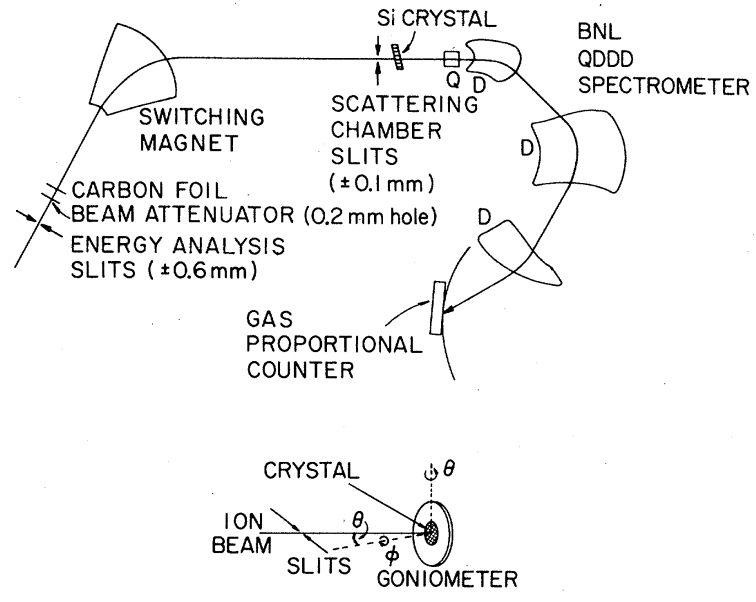

FIG. 1. Experimental setup.

the crystal in the beam and removed for each ion and charge state.

Momentum analysis of the beam after exiting the crystal was made with the BNL QDDD spectrometer with a $75-\mathrm{cm}$-long $\Delta E-E$ position sensitive proportional counter in the focal plane. The spectrometer slits were opened to the full solid angle $\left( \pm 3^{\circ}\right)$. Measurement of the total energy and energy loss, $\Delta E$, of the beam in the detector gas made it possible to discriminate against unwanted beams produced by the accelerator along with the desired beam. The spectrometer and focal plane detector were calibrated by observing the position of the beam (crystal out) peak for several magnetic fields in the spectrometer. The magnet was cycled at the beginning of the measurements, and whenever the magnetic field was decreased, in order to reduce errors in the calibration due to hysteresis in the magnet iron. To check for such errors the calibration was repeated periodically, and after large changes in magnetic field. All calibrations resulted in energy-loss determinations in agreement within $\pm 5 \mathrm{keV}$. The net instrumental resolution of the system including the accelerator ranged from $120 \mathrm{keV}$ for ${ }^{35} \mathrm{Cl}$ to $40 \mathrm{keV}$ for ${ }^{19} \mathrm{~F}$.

The thin crystalline silicon samples used in this work were prepared by a technique used for producing $\mathrm{x}$-ray lithography masks. ${ }^{13}$ Boron is diffused into a syton polished silicon wafer so that the doping level falls below $\sim 5 \times 10^{19} \mathrm{~cm}^{-3}$ at the depth corresponding to the desired sample thickness. An ethylene diamine pyrocatechol etch selectively removes material with doping levels below the above mentioned value. An annular mask placed around the edge of wafer during the etching process results in a ring of unetched silicon that supports the remaining boron doped thin crystal after the etch is completed. Channeling backscattering measurements have been performed on these samples and minimum yields indis- 
tinguishable from bulk undoped silicon were obtained. The sample thickness was determined experimentally by measuring the random energy loss of 5.47- $\mathrm{MeV}$ alpha particles from an ${ }^{241} \mathrm{Am}$ radioactive source. A value of $1.39 \mu \mathrm{m}$ was obtained with an uncertainty of $\sim 5 \%$.

Measured energy-loss spectra for the ${ }^{35} \mathrm{Cl}^{17+}$ beam are shown in Fig. 2 (as well as the energy distribution of the beam itself). The energy-loss values quoted in this article were obtained by fitting a straight line to the leading (low energy-loss) edge of such spectra and extrapolating the line to the energy-loss axis (see discussion below). The errors quoted present only the statistical uncertainties in extrapolating the leading edge and in determining the energy-loss calibration.

The three energy-loss spectra in Fig. 2 correspond to three orientations of the crystal axis with respect to the beam. The energy loss increases rapidly as the $\langle 110\rangle$ channel is tipped away from the beam direction. The crystal is oriented in the goniometer with the $\langle 110\rangle$ direction very close to the $\phi$ axis. Therefore, along the $\theta$ direction this increase occurs very rapidly (see Fig. 3). For the case of a ${ }^{32} \mathrm{~S}^{14+}$ beam a $10 \%$ error in $S / Q^{2}$ occurs for an angular misalignment of the channel by $0.02^{\circ}$. (" $Q$ " stands for the total charge of the penetrating ion.) Small errors in setting the goniometer can therefore lead to large systematic errors in the measured energy loss. The minimum energy loss was observed on a multichannel analyzer while setting the goniometer angles, and frequent checks of the accuracy of setting were made by taking partial angular distributions of the type shown in Fig. 3.

The leading edge energy loss $\Delta E$ is obtained by fitting a straight line to the experimental points and

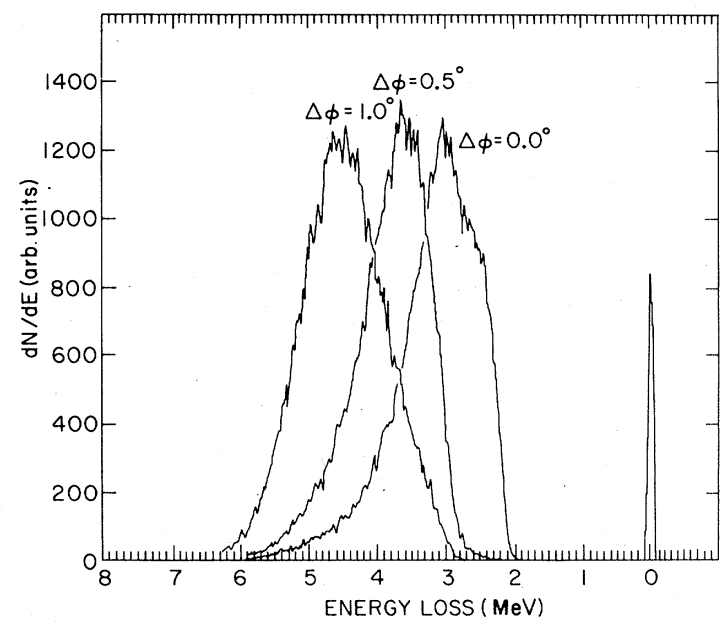

FIG. 2. Energy spectra for ${ }^{35} \mathrm{Cl}^{17+}$ beam showing change. in shape for different angular orientations.

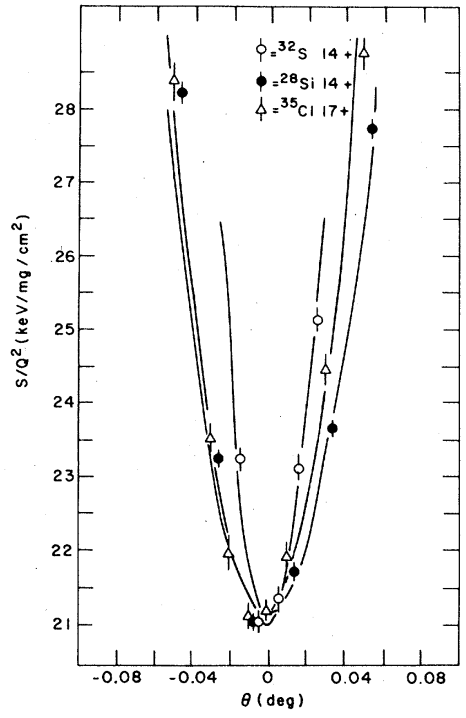

FIG. 3. Detailed angular dependence of $S / Q^{2}$ for ${ }^{32} \mathrm{~S}^{14+}$ and ${ }^{28} \mathrm{Si}^{14+}$ beams.

projecting this line to obtain the value of $\Delta E$ which corresponds to the loss of a particle traveling along the center of the crystal channel. It is however, a problem that the spectrum may be broadened by a number of mechanisms other than that stemming from the different trajectories of the ions. For example the spectrum is broadened by experimental energy resolution and this can be measured directly by passing the beam into the detection system to obtain the resolution function; the resolution function is easily unfolded from the data. For ions of higher $Z_{1}$ the energy losses become larger and the instrumental resolution correction becomes less important.

In addition to loss variations with ion trajectory in the channel, target thickness variations will introduce an additional broadening. For all sources of broadening listed up to this point, unfolding and leading edge extrapolation will give an accurate value for the best channeled energy loss. The leading edge extrapolation gives us, in the case that no other broadening is significant, the $\Delta E$ for the ion that passed through the least electron density and through the thinnest part of the crystal.

The very best channeled particles will not show a $\delta$-function energy spectrum. The real spectrum is broadened due to straggling. This straggling depends almost entirely upon close collisions with electrons and it can be calculated from electron-gas theory, using the midchannel electron density.

In our case, the important parameter is the ratio between the mean energy loss and the largest energy that can be lost in one collision with an electron. When this parameter is much greater than unity for any particular trajectory the statistical accumulation of 
individual loss events is large enough to guarantee a Guassian distribution of energy losses with a well defined mean and variance for each trajectory. Due to the low electron density at the center of a major channel, both of these quantities are expected to be a minimum for best channeled particles. Indeed the midchannel region for the silicon $\langle 110\rangle$ direction may be expected to have an exceptionally low electron density (say compared to a metal) due to the covalent nature of the silicon valence electron bonding. These bonds are far from the trajectory in question. Thus we estimate that systematic uncertainties in our leading edge and best-channeled energy losses are no greater than a few percent and uncertainty in quantities such as the $Z_{1}$ scaling over the range we have studied is even smaller. For $Z_{1} \leq 3-4$ these conclusions are invalid and closer attention must be paid to the straggling question. A reasonable approach to the problem is discussed in Ref. 3 .

\section{EXPERIMENTAL RESULTS}

A. Bare ions. For the bare nuclei, best channeled stopping powers, derived by the extrapolated leading edge method and divided by the squares of their respective nuclear charges are presented in Fig. 4. A least-squares analysis of the points has been made to determine coefficients for a constant term, a linear term, and a negative quadratic term for the $Z_{1}$ dependence of $S / Z_{1}^{2}$. The best fit occurs with no higher order terms and the result is $S / Z_{1}^{2}=21.2$ $\times(1 \pm 0.05) \mathrm{keV} / \mathrm{mg} \mathrm{cm}^{2}$; within these limits higher order terms in various combinations may exist. This question will be addressed further in the discussion section.

B. Clothed ions. As electrons are added to the ion, a point is reached where the added electron will be

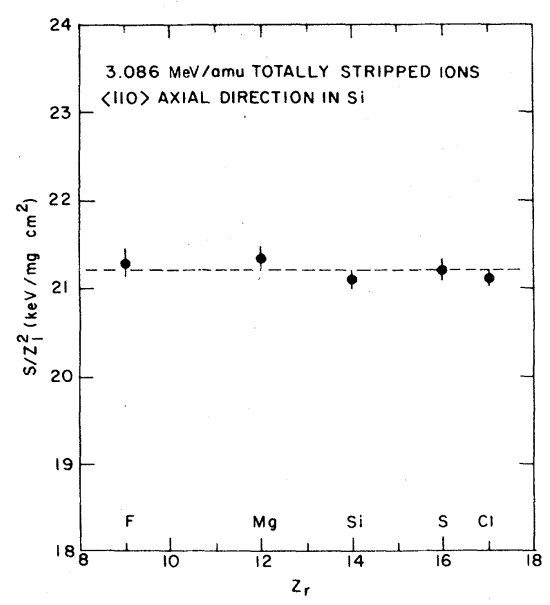

FIG. 4. $S / Z_{1}^{2}$ vs $Z_{1}$ for bare $\mathrm{F}, \mathrm{Mg}, \mathrm{Si}, \mathrm{S}$, and $\mathrm{Cl}$ ions.

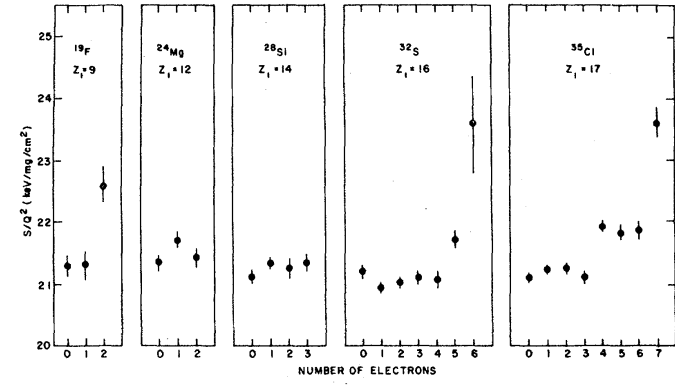

FIG. 5. Clothed and bare ion experimental results.

very weakly bound and it will occupy a large column of space around the path of the ion. At this point, even the best channeled particle will lose this last electron upon entering the crystal and will pass through the crystal with altered charge. If a few of these altered ions recapture an electron at the exit surface of the crystal they will then return to the initial (or entering) charge state and be recorded. When this finally occurs, the observed energy loss stops changing with input-exit charge and remains constant. The phenomenon of surface capture has been reported for channeled oxygen ions. ${ }^{14}$ In Table I we present the results obtained for both bare ions and clothed ions. The total charge of a clothed ion is indicated by " $Q$." Cases where the equal stopping power criterion shows a failure of charge state integrity are marked with an asterisk. The $L$-shell electrons of fluorine, magnesium, and silicon appear to be removed in the crystal and, in some cases, recaptured at the exit surface, but for higher $Z_{1}$, the electrons are more tightly bound and for these cases $L$-shell charge state integrity is maintained.

In Fig. 5 we display stopping powers $S / Q^{2}$ for clothed ions. Each series begins with the bare ion stopping power point and as electrons are added, nonlocality of the charge causes increases in the stopping power. As electrons are added the ionic charge becomes increasingly nonlocalized with an attendant increase in stopping power. Finally the point is reached where charge state integrity can no longer be sustained and no further data can be taken. Comparison of stopping power variations due to charge nonlocality with theoretical estimates will be made in the discussion section.

\section{DISCUSSION}

\section{A. Bare ion effects}

We begin our discussion by considering the energy-loss per unit path length for the bare ions. In the absence of channeling effects and assuming that heavy ions, of atomic number $Z_{1}$, pass through the 
TABLE I. Stopping powers for channeled heavy ions in $\mathrm{Si}\langle 110\rangle$.

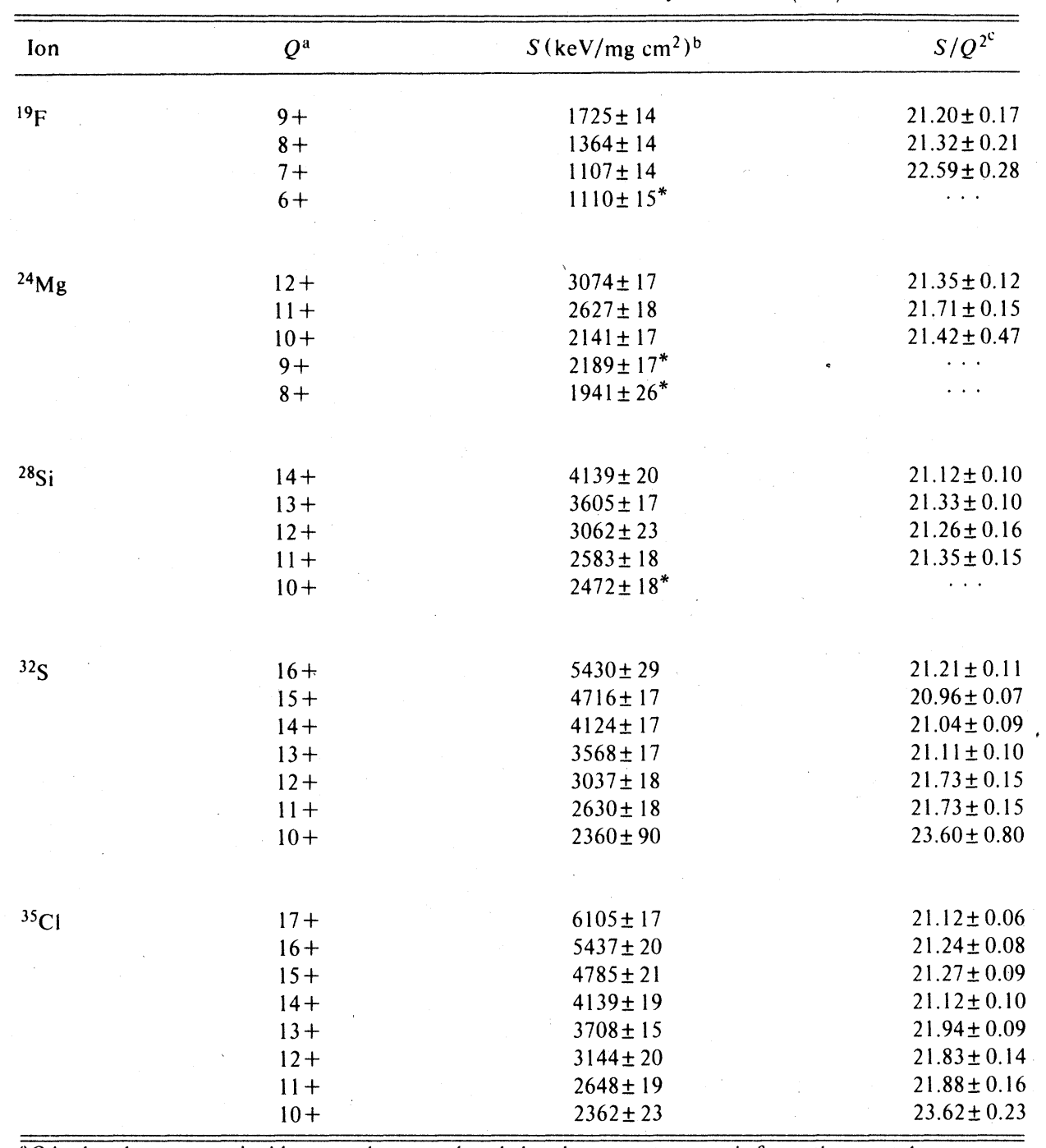

${ }^{a} Q$ is the charge state incident on the crystal and the charge state on exit from the crystal.

bNo value of $S / Q^{2}$ is given for charge states $Q=Q_{\text {in }}=Q_{\text {out }}$ where $q$ does not represent the

"frozen" charge in the channel (see text).

${ }^{c}$ Assuming crystal thickness of $0.324 \mathrm{mg} / \mathrm{cm}^{2}$ only errors in energy-loss measurement are included: systematic errors due to setting the crystal axis off channel are not included (see Fig. 3).

target totally stripped we expect

$\left\langle\frac{d E}{d x}\right)_{r}=\frac{4 \pi Z_{1}^{2} e^{4}}{m v^{2}} Z_{2} N\left(L_{0}+Z_{1} L_{1}+Z_{1}^{2} L_{2}+\ldots\right)$,

with

$$
\begin{aligned}
& L_{0}=\ln \frac{2 m v^{2}}{\hbar \bar{\omega}}, \\
& L_{1}=\frac{3 \pi}{2} \frac{e^{2} \tilde{\omega}}{m v^{3}} \ln \frac{m v^{2}}{\hbar \tilde{\omega}}, \\
& L_{2}=-\left(\frac{e^{2}}{\hbar v}\right)^{2} \sum_{n=1}^{\infty} \frac{1}{n\left[n^{2}+\left(e^{2} Z_{1} / \hbar v\right)^{2}\right]} .
\end{aligned}
$$

The subscript $(r)$ on the left-hand side of Eq. (1) indicates we are interested in stopping in a random direction. The ion velocity is $v$, electronic charge and mass are $e$ and $m$, and the target atomic number and density $Z_{2}$ and $N$, respectively.

The contribution to Eq. (1) from the term containing $L_{0}$ was deduced by Bethe ${ }^{15}$ via a quantal perturbation calculation to first order. Here $\hbar \bar{\omega}$ represents a particular weighted average of atomic excitation energies of the target. It is generally recognized that $L_{0}$ receives similar contributions from distant and close collisions with target electrons. [Actually included with $L_{0}$ in Eq. (2) should be a term representing 
shell effects. This term has no $Z_{1}$ dependence and for our case has a value $2-3 \%$ that of $L_{0}$ (Ref. 16).]

The additional contribution to Eq. (1) from the term containing $L_{1}$ has been discussed at some length in the literature recently $6,7,17,18$ and is generally known by the name of "Barkas effect" 5 after its discoverer. The form we have adopted for $L_{1}$ in Eq. (3) has been suggested by Lindhard ${ }^{8}$ who has called attention to the roughly equal role played by close and distant collisions in the Barkas effect. In all of the above the quantity $\kappa=Z_{1} e^{2} \hbar v$ is assumed much smaller than unity and as a result the term in Eq. (1) containing $L_{1}$ yields the only $Z_{1}^{3}$ dependence in the stopping. (We shall see that this point must be modified in the analysis of our data.) This part of the stopping may be looked upon as resulting from the inclusion of certain nonlinear features in the response of the atomic system to the penetrating ion. For $\kappa$ increasing towards unity it is reasonable that this contribution becomes more significant. Note that the "average" energy $\hbar \tilde{\omega}$ in $L_{1}$ is different from $\hbar \bar{\omega}$ previously discussed.

Finally as $\kappa$ increases towards one and beyond there are certain features of the Coulomb scattering of bound charges that may only be handled with great difficulty within the framework of a completely perturbation quantal calculation. Bloch ${ }^{19}$ has discussed this problem at some length in providing a connection formula between the regions $\kappa<<1$, where Bethe's formula applies, and $\kappa>>1$, where a classical calculation due to $\mathrm{Bohr}^{20}$ is expected to apply. Let it be sufficient here to note that the contribution to Eq. (1) from Eq. (4), the so-called "Bloch correction," arises from the inability of the quantal perturbation approach to adequately handle the small distance singular part of the Coulomb interaction. Collisions with atomic electrons specified by small angular momenta dominate this contribution which is therefore only associated with the close collision part of the stopping power. For small $\kappa, L_{2}$ is independent of $Z_{1}$ which accounts for the particular form of expansion in Eq. (1), with the Bloch correction yielding a $Z_{1}^{4}$ power correction to the stopping. ${ }^{8}$

In order to interpret our data we must modify the above discussion to incorporate the channeling effect and the large values of $\kappa(\kappa>\sim 1)$ associated with our experiments. Only when this is accomplished can it be shown that the processes associated with Barkas and Bloch corrections can play an important role in our experiments in spite of the nearly pure $Z_{1}^{2}$ dependence of the measured channeled stopping power.

For channeled particles several theories for the analogous contribution represented by $L_{0}$ in Eq. (1) have been discussed in the literature. ${ }^{21-25}$ The results generally may be applied when $\kappa<<1$, i.e., they are based on first-order quantal perturbation theory. These theories all predict therefore a $Z_{1}^{2}$ stopping dependence with a reduction factor of order 2-3 for well-channeled particles as compared to random direction penetration. A large part of this reduction factor, $\alpha$, may be heuristically explained as due to a reduction in close collision contributions to $L_{0}$ associated with the drop in electron density at the center of the channel. We set the best channeled energy-loss rate

$$
(d E / d x)_{\mathrm{ch}}^{\prime}=\alpha \frac{4 \pi e^{4}}{m v^{2}} Z_{1}^{2} Z_{2} N L_{0}
$$

and emphasize that this is the value expected from a "Bethe-like" first-order quantal calculation.

The channeling effects on the $Z_{1}^{3}$ contribution to the stopping may be estimated as follows. Since for random penetration roughly similar contributions from close and distant collisions are expected for both the Bethe and Barkas terms and since even the incremental contributions from a range of impact parameters is similar for both of these terms we suspect that at least roughly the $Z_{1}^{3}$ contribution to the stopping from the Barkas effect will be reduced for channeled particles again by the factor $\alpha$.

Finally the Bloch correction must be considered. Firstly, since this is a local effect we expect that for channeled particles it will depend on the average electron density on the path in the channel rather than the average total electron density $N Z_{2}$. Secondly, for the range of $\kappa$ values in our experiment $L_{2}$ is no longer independent of $Z_{1}$. In fact, $Z_{1}^{2} L_{2}$ is plotted in Fig. 6 and it is virtually linear in $Z_{1}$ for the region applicable to our experiments. Since the algebraic signs of the Barkas and Bloch corrections are opposite it is possible for these terms to cancel.

Summarizing, we may use Eq. (1) if we let

$$
\begin{aligned}
& L_{0} \Rightarrow \alpha L_{0}, \\
& L_{1} \Rightarrow \alpha L_{1}, \\
& L_{2} \Rightarrow \frac{\rho_{\mathrm{LOC}}}{Z_{2} N} L_{2},
\end{aligned}
$$

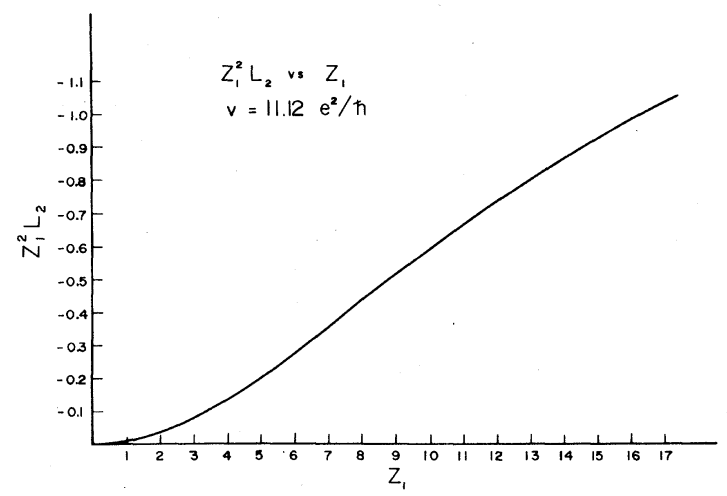

FIG. 6. Bloch correction $Z_{1}$ dependence for $v=11.12 e^{2} / \hbar$ 
where $\rho_{\text {LOC }}$ is the local average electron density on the channeled particle path, which for our considerations is at the center of the channel. If we further divide by $Z_{1}^{2}$ and the target density we obtain the quantity $S / Z_{1}^{2}$ we have used for our plots.

One thing is immediately clear. The constant value of $S / Z_{1}^{2}$ tells us that either (a) both the Bloch and Barkas effects are so small we have not detected them or (b) both may be large but they have virtually canceled each other out. We discuss these two possibilities shortly but first we note that regardless of our choice the only remaining contribution to the stopping is that from the first-order perturbation prediction. We thus obtain

$$
S / Z_{1}^{2}=21.2(1 \pm 0.05) \mathrm{keV} / \mathrm{mg} \mathrm{cm}^{2}
$$

Due to the small straggling corrections and small dechanneling rate here we expect this number to be quite reliable. Note that most of the uncertainty is associated with target thickness determination. This value for $S / Z_{1}^{2}$ is about $15 \%$ below the value calculated by Esbensen and Golovchenko ${ }^{25}$ and nearly $60 \%$ below that predicted by Dettmann. ${ }^{21}$ Furthermore Eq. (9) agrees well with the data of Eisen et al. ${ }^{26}$ for $\alpha$ particles of a velocity similar to that of our experiments.

Reasonable theoretical estimates of the magnitude of the $Z_{1}^{3}$ effects in our data require knowledge of $\rho_{\mathrm{LOC}}$ and $\hbar \tilde{\omega}$. It is not uncommon in channeling energy-loss theories to assume for $\rho_{\text {LOC }}$ in silicon channels a value obtained by using only valence electrons whose density is assumed uniform over the whole crystal. ${ }^{21,22}$ This gives $\rho_{\text {LoC }}=0.20$ electron $/ \AA^{3}$. Alternatively one may reconstruct charge densities from x-ray form factors such as those given by Doyle and Turner. ${ }^{27}$ For the $\langle 110\rangle$ channel in silicon we obtain $\rho_{\text {LOC }}=0.0672$ electron $/ \AA^{3}$. The Bloch correction is included for both of these charge densities in the plot in Fig. 7. The Barkas effect must clearly be included in the description if reasonable agreement between theory and experiment is to be obtained.

As mentioned earlier the contribution to $L_{1}$ from distant collisions has been calculated by Ashley et al. ${ }^{7}$ Using this work and assuming with Lindhard, ${ }^{8}$ that a nearly equal contribution also comes from close collisions and using Eq. (7) for channeled particles we find $\alpha L_{1} \approx 1.53 \times 10^{-2}$. For $\alpha$ we used the experimental value obtained as the ratio of our result in Eq. (9) to the random experimental value of $S / Z_{1}^{2}$ for $\mathrm{He}^{++}$in silicon (see Ref. $25 \mathrm{Fig} .1$ ). Use of the latter quantity is required since random $S / Z_{1}^{2}$ values cannot be obtained for the totally stripped ions in our experiments due to charge state fluctuations. Figure 7 shows both the first-order result for $S / Z_{1}^{2}$ incremented by only the resulting Barkas contribution and incremented by both Barkas and Bloch contributions (in curve labeled "total theory"). The Bloch contri-

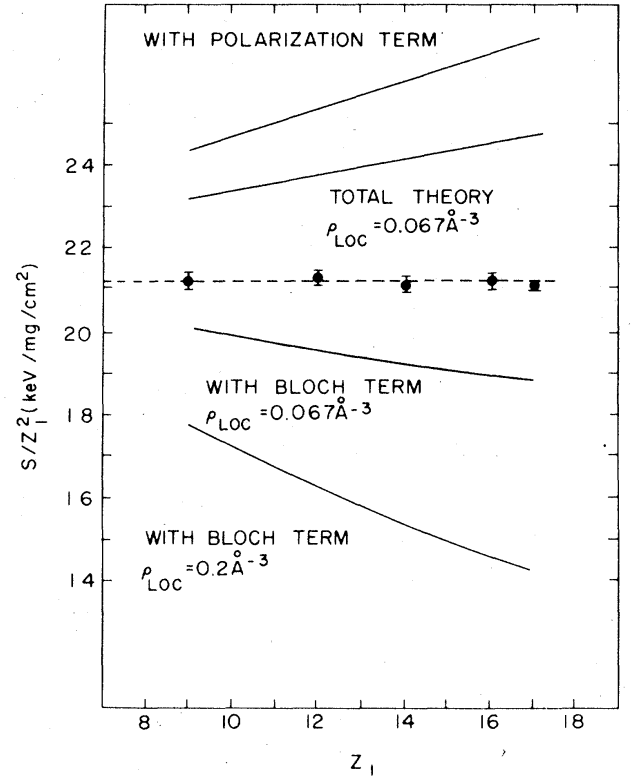

FIG. 7. Bare ion experimental results compared with estimate including Bloch correction and/or polarization term.

bution in the latter is taken using the 0.067 -electron/ $\AA^{3}$ center channel charge density. The resulting theoretical prediction is seen to be in poor agreement with the experimental points in the figure. We require substantially better cancellation of the Barkas and Bloch terms to improve the agreement. This can be achieved either by increasing $\rho_{\text {LOC }}$ or reducing $L_{1}$. Stimulated by this state of affairs we have sought further theoretical justification for the low value of $\rho_{\text {LOC }}$ adopted in the final comparison (Fig. 7). Selfconsistent-field pseudopotential calculations kindly performed by Dr. M. Schluter of Bell Laboratories indicate a value of $\rho_{\text {LOC }}=0.043$ electron $/ \AA^{3}$ with an estimated error of $25 \%$. Rather than adopt this, or any other number as a concrete value for $\rho_{\text {LOC }}$ we merely use it as further justification for discarding the uniform valence gas picture (with $\rho_{\mathrm{LOC}}=0.2$ electron/ $\AA^{3}$ ) mentioned earlier. It therefore appears that the Barkas contribution to our data is grossly overestimated by the current theory of the effect.

It should be realized that Eq. (3) for $L_{1}$ assumes that the excitation of the passing ion serves as a perturbation on the bound target electron motion. In particular the recoil of the electron during the course of the collision should be small compared to its atomic orbit size for the calculational scheme used in the derivation of $L_{1}$ to work. When this is the case the role of the target binding force is manifest through the appearance of $\tilde{\omega}$ in Eq. (3). The $Z_{1}^{3}$ effect is therefore a measure of the importance of binding forces in modifying the recoil of atomic electrons 
during the course of the collision. For the very large $Z_{1}$ and hence electronic recoils in our experiments target binding must play a diminished role in describing these recoils during a substantial part of the collision. Indeed, during these times it may well be more accurate to describe the evolution of the electronic states as being basically. Coulombic in the field of the $Z_{1}$ ion with the binding force of the target nucleus acting as a perturbation. Insofar as contributions to the Barkas effect are concerned, the perturbation plays a smaller role the higher $Z_{1}$ becomes and hence the Barkas effect disappears for high $Z_{1}$. It does not appear that the present structure of the theory of the Barkas effect can accommodate this possibility. We suggest, however, that the explanation for the reduced $Z_{1}^{3}$ contribution from polarization effects in our experiments lies in the above explanation.

\section{B. Clothed-ion effects}

We may now build upon our discussion of bare ion stopping to encompass those results where a few electrons are bound to the heavy projectile. We will now neglect all higher order $Z_{1}$ effects based on cancellation or smallness of the expected contributions. A first estimate of bound state screening effects on the stopping would seem to call on a perturbation calculation. Things are simplified even further if we concentrate on the difference in stopping power between a bare ion of charge $Z_{1}-1$ and a clothed ion of $Z_{1}$ with one bound electron in the ground state.
For this situation, excitation of the target electrons resulting from collisions with momentum transfers small compared to $\hbar / a_{k}$, where $a_{k}$ is the extension of the bound electron, may be expected to be identical for the two ions. On the other hand for large momentum transfer collisions, where the form factors for the two ions differ, there should be a significant difference in the stopping contribution. Moreover these large momentum transfers are associated with "close collisions" with electrons which suggests that they mainly depend upon the electron density on the track of the penetrating ions. Here this means the local electron density, $\rho_{\mathrm{LOC}}$, directly at the center of the channel.

A simple perturbation calculation for the difference in energy-loss rate between a singly clothed ion of atomic number $Z_{1}$ and a bare ion of $Z_{1}-1$ in an electron gas of charge density $\rho_{\mathrm{LOC}}$ gives for this quantity

$$
\begin{aligned}
& D_{H}\left(Z_{1}\right) \\
& \quad=\frac{4 \pi e^{4}}{m v^{2}} \bar{\rho}_{\text {LOC }} \int_{0}^{q} \frac{d q}{q}[1-F(q)]\left[2 Z_{1}-1-F(q)\right],
\end{aligned}
$$

where $q=2 m v / \hbar$ and

$$
F(q)=\left(1+\frac{q^{2} a_{0}^{2}}{4 Z_{1}^{2}}\right)^{-2}
$$

is the atomic form factor for the electron in the hydrogenlike ion. $a_{0}=\hbar^{2} / m e^{2}$ is the Bohr radius.

Using Eq. (11) in Eq. (10) integration yields

$$
D_{H}=\frac{4 \pi e^{4}}{m v^{2}} \bar{\rho}_{\mathrm{LOC}}\left(\frac{\left(2 Z_{1}-1\right)}{2}\left[\ln F^{-1 / 2}(q)-F^{1 / 2}(q)+1\right]-\frac{1}{6}\left[1-F^{3 / 2}(q)\right]-\frac{1}{4}[1-F(q)]\right) .
$$

An analogous result for $\mathrm{He}$-like ions exists with

$$
D_{\mathrm{He}}\left(Z_{1}\right)=\frac{4 \pi e^{4}}{m v^{2}} \bar{\rho}_{\mathrm{LOC}}\left\{\left(2 Z_{1}-2\right)\left[\ln G^{-1 / 2}(q)-G^{1 / 2}(q)+1\right]-\frac{1}{6}\left[1-G^{3 / 2}(q)\right]-\frac{1}{4}[1-G(q)]\right\},
$$

where

$$
G(q)=\left[1+\left(\frac{q a_{0}}{2 Z_{1}-5 / 8}\right)^{2}\right]^{-2}
$$

Here $D_{\mathrm{He}}$ is the difference in energy loss rate between a bare ion of atomic number $Z_{1}-2$ and a two electron clothed ion of atomic number $Z_{1}$. The electrons are assumed to be in ground state heliumlike orbitals represented by the common variational best guess for the product hydrogen wave function.

The predictions of these formulas for the case of fluorine, magnesium, and silicon in our data are shown in Fig. 8. The value of $\rho_{\text {LOC }}=0.067$ electron $/ \AA^{3}$, was used in these estimates for the average center channel charge density. Agreement seems reasonable enough to indicate that the simple bound electron screening contributes significantly to the observed effects. As $Z_{1}$ increases these effects become diluted for the first two bound electrons due to the decreasing size of the atom orbits. Furthermore for increasing $Z_{1}$ the quantal perturbation technique upon which these predictions are based becomes less justifiable. A classical calculation would yield smaller effects yet. For lower $Z_{1}$ these screening effects get larger and we have applied the above formulas also to the data of Datz et al. using a [111] Au center channel charge density of 0.19 electron $/ \AA^{3}$. This value was obtained from a full augmented plane wave (APW) band-structure calculation. Figure $9 \mathrm{com}$ pares calculations with experimental results and agreement is again quite reasonable. 


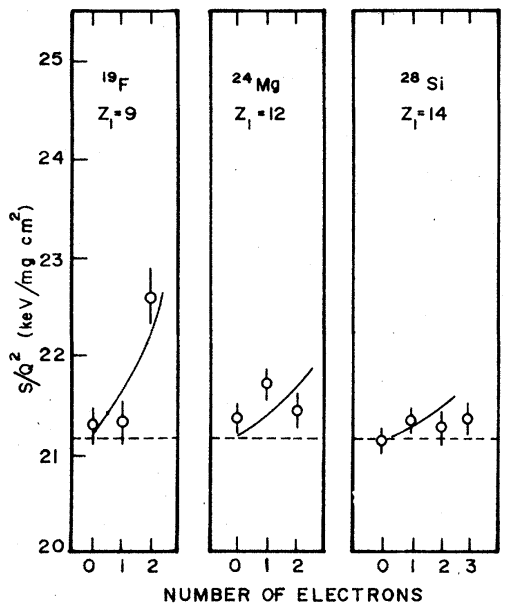

FIG. 8. Screened ion data for F, Mg, and Si compared with theoretical estimates (solid line).

Before closing this section we finally note that Besenbacher et $a l .{ }^{28}$ have recently observed an effect analogous to the above through a comparison of $\mathrm{H}^{+}$ and $\mathrm{He}^{+}$energy-loss straggling data.

\section{CONCLUSION}

Using the channeling effect to maintain charge state integrity for bare and partially clothed ions we have presented comprehensive energy-loss data ranging from $Z_{1}=9$ to 17 . Our results show a complete absence of the higher order $Z_{1}$ effects that might be expected at this velocity. This seems comprehensible within the framework of current thinking only if both Barkas and Bloch corrections are small, or they are

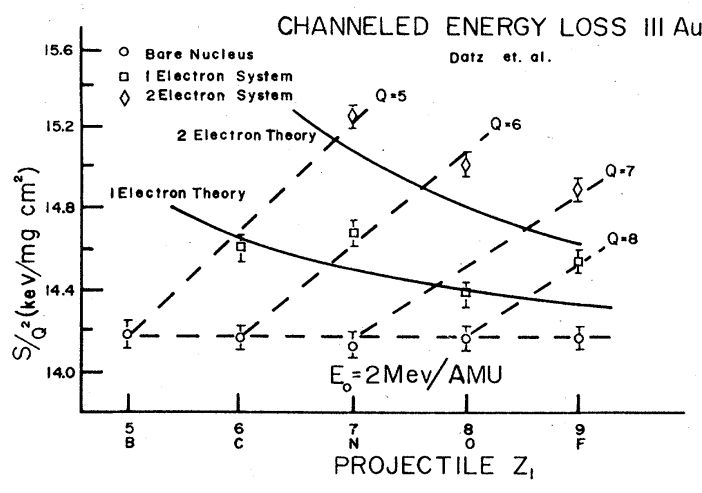

FIG. 9. Data of Datz et al. (Ref. 11) compared with theoretical estimates (solid line).

sizable but cancel. Simple estimates suggest the latter. Screening effects due to bound charges on the projectile are also observed and are found to agree with simple estimates. Here again a sensitivity to local charge density on the particle path is indicated. It would certainly appear that observed energy-loss differences for different ions of the same charge state tends to deprive effective charge state approaches of deep physical meaning.

Of all our results, the near absence of higher order $Z_{1}$ effects is the most intriguing. Our current thinking is that this result is just an "accident." This conclusion would be dramatically altered if the minimum $\langle 110\rangle$ charge density was significantly below $0.067 / \AA^{3}$. If this were the case we would simply conclude that both Bloch and Barkas corrections were beyond the reach of our experiments. We would then be at a loss to explain the fluorine screened ion results.
"Present address: Institute of Physics, Aarhus University, Aarhus C, Denmark.

${ }^{1}$ N. Bohr, K. Dan. Vidensk. Selsk. Mat.-Fys. Medd. 18 , No. 8 (1948).

${ }^{2}$ V. Fano, Annu. Rev. Nucl. Sci. 13, 1 (1963).

${ }^{3}$ H. Esbensen, O. Fich, J. A. Golovchenko, S. Madsen, H. Nielsen, H. Schiøtt, E. Uggerhøj, C. Vraast-Thomsen, G. Charpak, S. Majewski, G. Odyniec, G. Petersen, F. Sauli, J. P. Ponpon, and P. Siffert, Phys. Rev. B 18, 1039 (1978).

${ }^{4}$ M. D. Brown and C. D. Moak, Phys. Rev. B $\underline{6}, 90$ (1972).

${ }^{5}$ W. H. Barkas, N. J. Dyer, and H. H. Heckman, Phys. Rev. Lett. 11, 26 (1963). See also H. H. Heckman and P. J. Lindstrom, Phys. Rev. Lett. 22, 871 (1969).

${ }^{6}$ J. D. Jackson and R. L. McCarthy, Phys. Rev. B $\underline{6}, 4131$ (1972).

7J. C. Ashley, R. H. Ritchie, and W. Brandt, Phys. Rev. B $\underline{5}$,
2393 (1972): S. H. Morgan and C. C. Sung. Phys. Rev. A 20), $818(1979)$.

8J. Lindhard, Nucl. Instrum. Methods 132, 1 (1976).

${ }^{9}$ H. H. Andersen, J. F. Bak, H. Knudsen, P. Møller Petersen, and B. R. Nielsen, Nucl. Instrum. Methods 140, 537 (1977). See also H. H. Andersen, J. F. Bak, H. Knudsen, and B. R. Nielsen, Phys. Rev. A 16, 1929 (1977).

${ }^{10}$ C. D. Moak, S. Datz, B. R. Appleton, J. A. Biggerstaff, M. D. Brown, H. F. Krause, and T. S. Noggle, Phys. Rev. B 10, 2681 (1974).

${ }^{11}$ S. Datz, J. Gomez del Campo, P. F. Dittner, P. D. Miller, and J. A. Biggerstaff, Phys. Rev. Lett. 38,1145 (1977).

${ }^{12}$ S. P. Ahlen, Phys. Rev. Lett. 39, 1398 (1977).

${ }^{13}$ C. J. Schmidt, P. V. Lenzo, and E. G. Spencer, J. Appl. Phys. 46, 4080 (1975).

${ }^{14}$ B. R. Appleton, R. H. Ritchie, J. A. Biggerstaff, T. S. Nog- 
gle, S. Datz, C. D. Moak, H. Verbeek, and V. N. Neelavathi, Phys. Rev. B 19, 4347 (1979).

${ }^{15}$ H. A. Bethe, Ann. Phys. (Leipzig) 5, 325 (1930).

${ }^{16}$ E. Bonderup, K. Dan. Vidensk. Selsk. Mat.-Fys. Medd. 35, No. 17 (1967).

${ }^{17}$ J. C. Ashley, R. H. Ritchie, and W. Brandt, Phys. Rev. A 8,2402 (1973).

${ }^{18}$ K. W. Hill and E. Merzbacher, Phys. Rev. A $\underline{9}, 156$ (1974).

${ }^{19} \mathrm{~F}$. Bloch, Ann. Phys. (Leipzig) 16, 285 (1933).

${ }^{20}$ N. Bohr, Philos. Mag. 25, 10 (1913).

${ }^{21}$ K. Dettmann, Z. Phys. A 272, 227 (1975).

${ }^{22}$ B. R. Appleton, C. Erginsoy, and W. M. Gibson, Phys.
Rev. 161,330 (1967).

${ }^{23}$ F. Bonsignori and A. Desalvo, J. Phys. Chem. Soc. 31, 2191 (1970).

${ }^{24}$ F. F. Komarov and M. A. Kumakhov, Radiat. Eff. 22, 1 (1974).

${ }^{25} \mathrm{H}$. Esbensen and J. A. Golovchenko, Nucl. Phys. A $\underline{298}$, 382 (1978).

${ }^{26} \mathrm{~F}$. H. Eisen, G. J. Clark, J. Bottinger, and J. M. Poate, Radiat. Eff. 13, 93 (1972).

${ }^{27}$ P. A. Doyle and P. S. Turner, Acta Crystallogr. Sect. A 24, 390 (1968).

${ }^{28} \mathrm{~F}$. Besenbacher, J. U. Andersen, and E. Bonderup, Nucl. Instrum. Methods 168, 1 (1980). 